\title{
Perdas na colheita de tomate industrial em função da regulagem da colhedora ${ }^{1}$
}

\author{
João Paulo Barreto Cunha², Túlio de Almeida Machado³, Fábio Lúcio Santos ${ }^{4}$, Laysla Moraes Coelho ${ }^{5}$
}

\begin{abstract}
Losses in industrial tomato

harvesting according to harvester setting

Losses in the mechanical harvesting of industrial tomatoes, depending on the levels reached, may considerably reduce the yield of the planted areas. This study aimed to evaluate the effect of settings of the rotary separation system on losses observed during the mechanized harvesting process. The statistical design was completely randomized, in a factorial scheme with four replications, where each plot consisted of the combination of three rotation levels (6 rpm, $24 \mathrm{rpm}$ and $18 \mathrm{rpm}$ ) with three vibration frequencies $(0.83 \mathrm{~Hz}, 2.50 \mathrm{~Hz}$ and $4.17 \mathrm{~Hz})$ from the separation system. The recorded losses were divided into branch losses, fruit losses on the soil and total losses. Sequential and control charts for individual values and variable ranges composed by the upper and lower limits of control and average were used as a tool of statistical process control. The results showed that the total losses incurred are outside the control limits and acceptable standards for industrial tomato crops. The use of higher levels of rotation and vibration in the harvester separation system provided a higher harvest efficiency.
\end{abstract}

KEY-WORDS: Solanum lycopersicum L.; control charts; mechanical harvesting.

\section{INTRODUÇÃO}

O tomate (Solanum lycopersicum L.) é uma hortaliça originária da América do Sul, sendo considerada a segunda hortaliça em volume mundial de produção e consumo, bem próximos aos da batata, que apresenta os maiores registros. Dessa maneira, segundo Ubierna et al. (2010), atualmente, o setor de tomate para processamento passa a ter reconhecimento mundial, devido, principalmente, à sua grande importância econômica, a qual possui como base as grandes indústrias de atomatados espalhadas

\section{RESUMO}

As perdas na colheita mecanizada de tomate industrial, dependendo dos níveis atingidos, podem reduzir consideravelmente a produtividade das áreas plantadas. Dentro desse contexto, este estudo objetivou avaliar o efeito da regulagem do sistema de separação rotativo nas perdas observadas durante a realização da colheita mecanizada. O delineamento estatístico utilizado foi inteiramente casualizado, em esquema fatorial, com quatro repetições, onde cada parcela consistiu da combinação de três níveis de rotação (6 rpm, 12 rpm e $18 \mathrm{rpm})$ e três frequências de vibração $(0,83 \mathrm{~Hz}, 2,50 \mathrm{~Hz}$ e $4,17 \mathrm{~Hz}$ ) do sistema de separação. As perdas contabilizadas foram divididas em perdas nas ramas, perdas de frutos no solo e perdas totais. Cartas sequenciais e cartas de controle para valores individuais e de amplitude móveis, compostas pelos limites superior e inferior de controle e média, foram utilizadas como ferramentas de controle estatístico de processo. Os resultados indicaram que as perdas totais ocasionadas encontram-se fora dos limites de controle e dos padrões aceitáveis para a cultura do tomate industrial. A adoção de maiores vibrações e rotações do sistema de separação da colhedora proporcionaram maior eficiência de colheita.

PALAVRAS-CHAVE: Solanum lycopersicum L.; cartas de controle; colheita mecanizada.

em todos os grandes centros consumidores.

A mecanização do processo de colheita do tomate industrial vem proporcionando melhores resultados em todo o mundo, com o aumento da capacidade operacional. No Brasil, trata-se de um processo recente, e que ganhou importância a partir da década de 1990, coincidindo com a expansão da cultura do tomate no Estado de Goiás, sendo praticado por grandes produtores, os quais, sob condições adequadas, vêm utilizando essa tecnologia, visando ao transplantio em áreas extensas e ao aumento da praticidade da colheita (Cortez et al. 2002).

1. Trabalho recebido em out./2013 e aceito para publicação em out./2014 ( $\mathrm{n}^{\mathrm{o}}$ registro: PAT 26892).

2. Universidade Federal de Lavras (UFLa), Departamento de Engenharia, Lavras, MG, Brasil. E-mail: bcunha_2@hotmail.com.

3. Instituto Federal Goiano (IFGoiano), Campus Morrinhos, Morrinhos, GO, Brasil.E-mail: machado.tulio@gmail.com.

4. Universidade Federal de Viçosa (UFV), Departamento de Engenharia Agrícola, Viçosa, MG, Brasil.E-mail: fabio.ls@ufv.br. 5. Universidade Federal de Goiás (UFG), Escola de Agronomia, Goiânia, GO, Brasil. E-mail: layslamc@hotmail.com. 
A colheita mecanizada do tomate vem permitindo a redução de custos por unidade $\left(\mathrm{R} \$ \mathrm{t}^{-1}\right)$ e de mão de obra, por apresentar maior confiabilidade nas operações e, consequentemente, maior custo/ benefício (Jarén et al. 2007).

De modo geral, as colhedoras empregadas são de origem italiana ou americana, desenvolvidas na década de 1970, e que, durante as décadas seguintes, passaram por grande evolução, tornando-se autopropelidas e mais eficientes no sistema de trilha e seleção dos frutos. Segundo Arazuri et al. (2007), as principais diferenças entre os modelos de colhedoras estão basicamente relacionadas ao sistema de destacamento dos frutos, posição das correias de seleção e unidade de descarga do produto.

Dois sistemas de destacamento sobressaem-se: o sistema de cintas e o rotativo. De acordo com Arazuri et al. (2010), a tendência dos modelos mais novos de colhedoras é a utilização de sistemas rotativos, que, mesmo apresentando baixas rotações na sua operação, apresentam vantagens, como o tamanho reduzido, menor necessidade de energia para separação dos frutos e menor ruído.

Por se tratar de um processo ainda novo no Brasil, as perdas estão relacionadas às questões de manejo, principalmente pela utilização de equipamentos nem sempre adaptados à nossa realidade. No processo de colheita, se dá o grande problema de sistematização das áreas utilizadas para o cultivo, visto que a colhedora não se adapta a terrenos irregulares, ocasionando, assim, maior número de impurezas na carga. Machado et al. (2011) destacam que a ação dos dentes da plataforma, dessa maneira, será prejudicada, podendo levar uma quantidade grande de impurezas para os diversos mecanismos da máquina, danificando o equipamento e reduzindo a qualidade do produto colhido.

Trabalhos de avaliação de perdas causadas pela colhedora de tomate e seus mecanismos são raros. Essas perdas, quando monitoradas, podem servir como indicadores da qualidade do processo de colheita, pois, como constataram Ferreira et al. (2006), sempre foi difícil o processo de mensurar as perdas nessa etapa e, no caso da cultura do tomate, há mais uma preocupação, por se tratar de produto bastante sensível e que envolve alto custo horário com máquinas e mão de obra.

O controle estatístico de processo (CEP) é uma ferramenta que permite melhor compreensão das operações mecanizadas, principalmente do processo de colheita, devido aos bons resultados obtidos, visto que busca a melhoria do processo pela redução da variabilidade (Toledo et al. 2008).

Compangnon et al. (2012), na comparação das perdas em campo, utilizando uma colhedora axial de soja e a saída fornecida pelo monitor de perdas, durante a colheita em diferentes períodos do dia, verificaram, por meio de cartas de controle, que houve, no período noturno, uma maior coincidência entre os níveis obtidos no sensor de separação e as perdas medidas em campo na colheita da soja, em relação ao diurno.

Avaliando a qualidade de operação da colheita mecanizada de feijão cultivado sob plantio convencional e plantio direto, Silva et al. (2013) verificaram efeito de variáveis relacionadas à qualidade da colheita e características operacionais da máquina, por meio de controle estatístico de processo. As cartas de controle permitiram identificar as variáveis com condições de manter a qualidade da operação de colheita, tanto no preparo convencional de solo quanto no plantio direto.

Diante do pressuposto de que as regulagens das colhedoras afetam diretamente a qualidade da operação, o presente trabalho objetivou determinar as perdas quantitativas, em função de diferentes vibrações e rotações do sistema de separação da máquina, por meio do controle estatístico de processo (CEP).

\section{MATERIAL E MÉTODOS}

O estudo foi realizado no município de Morrinhos (GO), em Latossolo Vermelho distrófico, sendo utilizada a cultivar de tomate Lycopersicon esculentum Mill. HEINZ 9553, em sistema de transplantio direto.

O processo de colheita aconteceu em setembro de 2012, aos 125 dias após o transplantio semimecanizado, sendo utilizada, para a avaliação das perdas, uma colhedora autopropelida da marca GUARESI, modelo G-89/93 MS 40", com motor FIAT-Iveco $129 \mathrm{~kW}$ e plataforma de recolhimento com espaçamento de $1,5 \mathrm{~m}$, flutuante e dotada de selecionador eletrônico de frutos verdes e torrões. Durante os ensaios, a colhedora foi utilizada com a rotação do motor em 1.900 rpm e velocidade média operacional de $1,14 \mathrm{~m} \mathrm{~s}^{-1}$.

No momento da colheita, o solo encontrava-se com teor médio de água de 18,4\% (Embrapa 1997). A verificação desse fator é de suma importância, 
pois afeta diretamente a eficiência da operação de colheita do tomate.

O delineamento experimental adotado foi inteiramente casualizado, em esquema fatorial, com quatro repetições, onde cada parcela consistiu da combinação de três níveis de rotação $(6 \mathrm{rpm}, 12 \mathrm{rpm}$ e $18 \mathrm{rpm})$ e três frequências de vibração $(0,83 \mathrm{~Hz}$, $2,50 \mathrm{~Hz}$ e 4,17 Hz) do sistema de separação. Dessa forma, a área amostrada foi constituída de 36 parcelas, equidistantes em $70 \mathrm{~m}$, selecionadas sequencialmente no início da colheita.

Para a quantificação das perdas, foi utilizado um gabarito com área de 2,5 $\mathrm{m}^{2}$ (1,66 $\mathrm{m}$ x 1,5 m), no qual, após a passada da colhedora, foram coletados, em cada um dos pontos, os materiais necessários para as avaliações das perdas propostas no presente trabalho. Após ser recolhido, o material foi ensacado, identificado e pesado em balança digital (modelo AD2000) com precisão de $0,01 \mathrm{~g}$.

As perdas contabilizadas foram divididas em perdas nas ramas e perdas de frutos no solo. As perdas nas ramas constituíram-se pelos frutos que não se destacaram das ramas após a passagem pelo sistema de trilha. As perdas de frutos no solo consistiram do somatório de frutos não recolhidos pela colhedora $\mathrm{e}$ pelos frutos destacados das ramas e caídos da colhedora pelos vãos das esteiras. As perdas naturais não foram contabilizadas nesse processo, já que, mesmo com frutos destacados das ramas, há, ainda, a possibilidade de recolhimento dos mesmos, por parte da colhedora. As perdas totais foram contabilizadas pelo somatório das perdas nas ramas e no solo.

Utilizando-se estatística descritiva, foi possível a visualização geral do comportamento dos dados. Dessa forma, foram calculados a média aritmética, mediana, valor máximo e mínimo, desvio-padrão e coeficientes de variação (CV), assimetria (cs) e curtose (ck). A averiguação da normalidade dos dados obtidos foi realizada pelo teste Shapiro-Wilk, sendo utilizadas, como métodos estatísticos, cartas de controle, para cada variável. As cartas de controle e amplitude apresentam, como linha central, a média geral, amplitude média e limites superior (LSC) e inferior de controle (LIC), calculados com base no desvio-padrão das variáveis (para LSC $=3 \sigma$ e para $\mathrm{LIC}=-3 \sigma$, quando maior que zero).

As perdas, em relação às diferentes regulagens da máquina, foram comparadas com limites aceitáveis para a perda total em colheita mecanizada de tomate, sendo limitadas em até $6 \%$ (Machado et al. 2011).

\section{RESULTADOS E DISCUSSÃO}

A Tabela 1 fornece os parâmetros da análise descritiva dos dados obtidos, os quais indicam que ocorreu alta variabilidade nos valores encontrados para as diferentes perdas estudadas, uma vez que a variabilidade de um atributo está diretamente relacionada com a magnitude do seu coeficiente de variação (Freddi et al. 2006). As perdas totais (Ptot), perdas de frutos no solo (Psol) e perdas de frutos não destacados (Pram) apresentaram distribuição assimétrica e coeficientes de curtose e assimetria positivos, o que vem a explicar a maioria dos valores abaixo da média. Outro fato explicado pelo coeficiente de curtose positivo é que, mesmo considerado em certas citações elevado, permitiu demonstrar que os valores de perdas tenderam a se concentrarem em torno da média.

As perdas totais (Ptot) se mostraram fora de controle, ou seja, distribuídas de maneira instável (Figura 1). Verificou-se que a amplitude entre os pontos 1-2 ultrapassou o limite superior de controle (LSC), indicando a diferença de configuração do mecanismo de separação utilizado, ou seja, a amplitude nas perdas ocorreu devido à utilização de uma vibração muito diferente no ponto 2. Com relação ao ponto 26 , o mesmo atingiu perdas totais superiores ao LSC, indicando, assim, que causas especiais interferiram no processo.

Tabela 1. Análise estatística descritiva para percentagem de perdas de frutos não destacados (Pram), perdas de frutos no solo (Psol) e perdas totais (Ptot) (Morrinhos, GO, 2012).

\begin{tabular}{|c|c|c|c|c|c|c|c|c|c|}
\hline \multirow{2}{*}{ Variável } & \multirow{2}{*}{ Média } & \multirow{2}{*}{ Mediana } & \multicolumn{2}{|c|}{ Valor } & \multirow{2}{*}{$\begin{array}{l}\text { Desvio- } \\
\text { padrão }\end{array}$} & \multicolumn{3}{|c|}{ Coeficiente } & \multirow{2}{*}{ Teste } \\
\hline & & & Máximo & Mínimo & & CV (\%) & $\mathrm{Ck}$ & $\mathrm{Cs}$ & \\
\hline Pram & 4,72 & 3,96 & 11,82 & 1,230 & 2,71 & 57,35 & 0,149 & 0,874 & A \\
\hline Psol & 2,79 & 2,06 & 10,01 & 0,884 & 2,06 & 73,58 & 4,090 & 2,050 & A \\
\hline Ptot & 7,52 & 6,40 & 18,94 & 2,950 & 3,90 & 51,92 & 0,395 & 0,901 & $\mathrm{~A}$ \\
\hline
\end{tabular}

Ck: coeficiente de curtose; Cs: coeficiente de assimetria; A: distribuição assimétrica pelo teste de Shapiro-Wilk. 

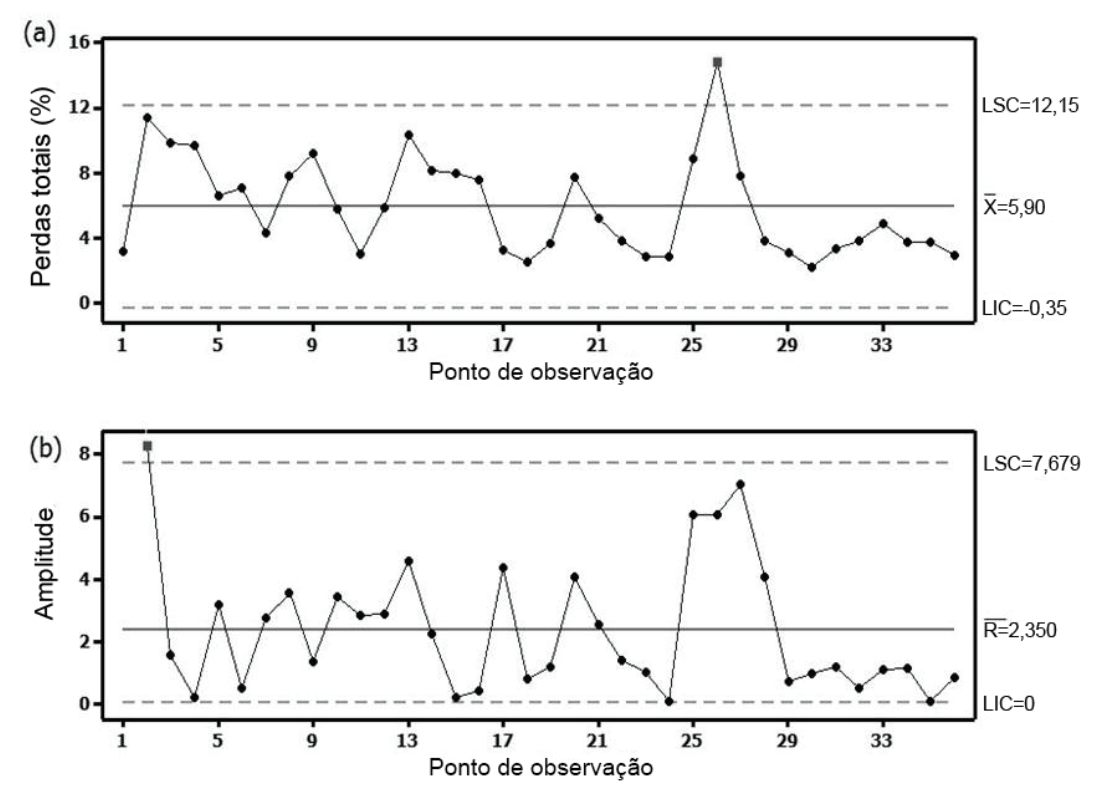

Figura 1. Carta de controle para perdas totais (\%) (a) e carta de amplitude móvel (b) (Morrinhos, GO, 2012). LSC: limite superior de controle; LIC: limite inferior de controle; X e Amplitude: média.

Segundo Chioderoli et al. (2012), a variação de um processo pode ser ocasionada por causas comuns e especiais. No caso das causas comuns, as mesmas não podem ser evitadas. Em contrapartida, as causas especiais, em operações agrícolas, podem estar relacionadas a diversos fatores, como experiência do operador, condições do solo e, principalmente, regulagens da colhedora, o que corrobora os resultados obtidos, nos quais, em ambos os pontos, a máquina trabalhava com vibrações e rotações menores, fato que propiciou aumento nas perdas e, consequentemente, menor eficiência de colheita.

Quando relacionadas as vibrações utilizadas no presente estudo, o processo permaneceu estável, com a distribuição dos valores dentro dos limites aceitáveis para as menores vibrações. Em contrapartida, para a vibração de $4,7 \mathrm{~Hz}$, o processo apresentou instabilidade, visto que o ponto 25 se encontra acima do LSC, podendo este valor estar relacionado à quantidade de material a ser trilhado pelo mecanismo de separação da máquina, o que pode ter contribuído para esse resultado (Figura 2a).

De acordo com Oliveira et al. (2007), o uso da vibração e/ou o impacto mostram-se como métodos eficientes para a colheita de diversos produtos agrícolas. Assim, diferentes tipos de colhedoras foram desenvolvidas segundo esse princípio, como as de café, citros, azeitona e o sistema de separação da colhedora de tomate.
No caso das colhedoras de citros, são utilizadas vibrações próximas das estudadas no presente trabalho, o que permitiu uma melhor comparação dos resultados. Diversos autores, como Whitney (1998) e Peterson (1998), avaliando a colheita mecanizada de laranja, verificaram, durante os testes, que a utilização de uma frequência de vibração de $5 \mathrm{~Hz}$ permitiu a colheita de frutos com qualidade semelhante à da colheita manual e eficiência de derriça de $80-90 \%$, resultados também obtidos no presente ensaio.

Nesse cenário, quando utilizadas as duas maiores vibrações, verificou-se que as perdas totais foram menores e dentro de limites aceitáveis (Machado et al. 2011). Diferentemente dessa situação, constatou-se que, quando utilizada a menor vibração $(0,83 \mathrm{~Hz})$, houve perdas totais médias de, aproximadamente, $10,7 \%$, valor acima do aceitável.

Tatlidil et al. (2005), avaliando as perdas ocasionadas no período de colheita mecanizada de tomate, observaram valores de perdas totais de $5,1-9,8 \%$. Os mesmos autores citam a necessidade de um controle bem feito de todos os parâmetros de produção, por parte dos produtores, onde a regulagem adequada das colhedoras e o transporte eficiente do produto, quando bem monitorados, podem minimizar as perdas.

Para as perdas relacionadas aos frutos não destacados pela máquina (Pram), verificou-se comportamento estável na distribuição dos valores, com 
os mesmos dentro dos limites aceitáveis de controle inferior e superior, sendo possível observar que, quando utilizada a menor vibração, em média, os valores apresentaram-se acima do limite aceitável de 6\% (Figura 3a). Para essa variável, a média geral de perdas foi de $4,58 \%$, correspondendo a, aproximadamente, $63 \%$ das perdas totais obtidas, confirmando que as perdas relacionadas aos frutos não destacados é o fator que exerce maior influência nas perdas totais, sendo necessária escolha criteriosa da vibração do sistema de separação.

Para as perdas no solo (Psol), o processo se comportou de maneira estável, quando utilizada a menor rotação (Figura 4a). Em contrapartida, houve
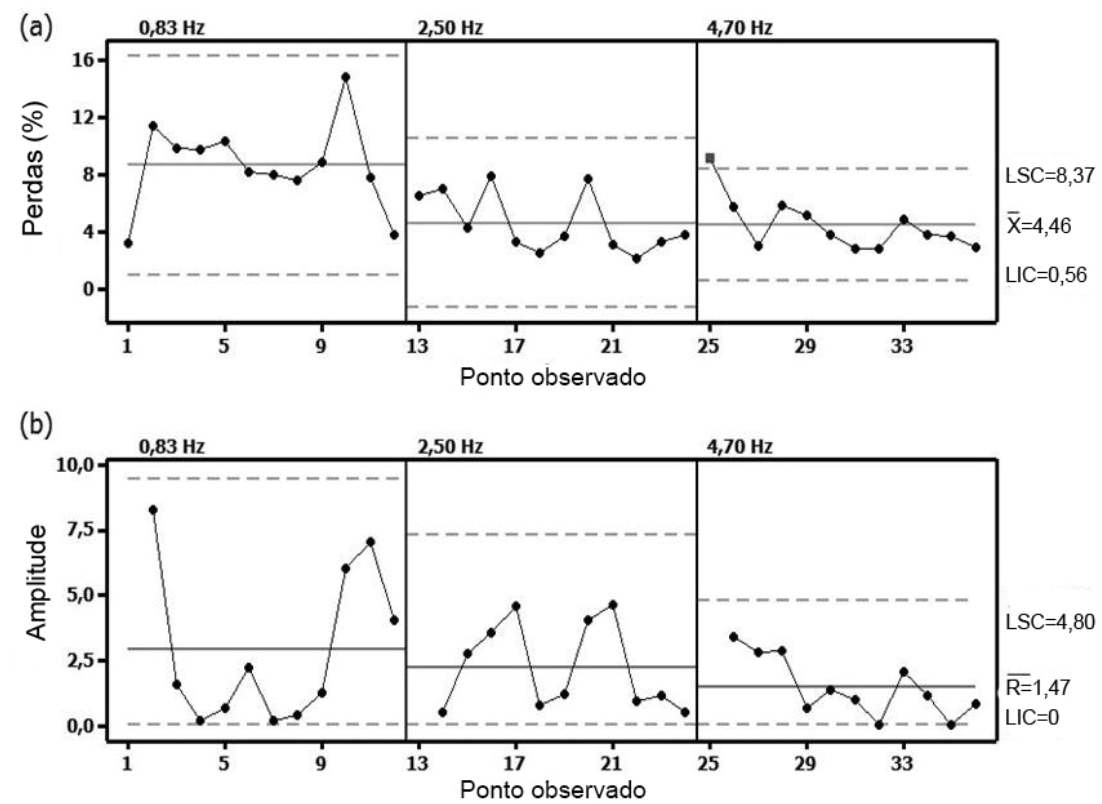

Figura 2. Carta de controle para perdas totais (\%) relacionadas à vibração (a) e carta de amplitude móvel (b) (Morrinhos, GO, 2012). LSC: limite superior de controle; LIC: limite inferior de controle; X e Amplitude: média.
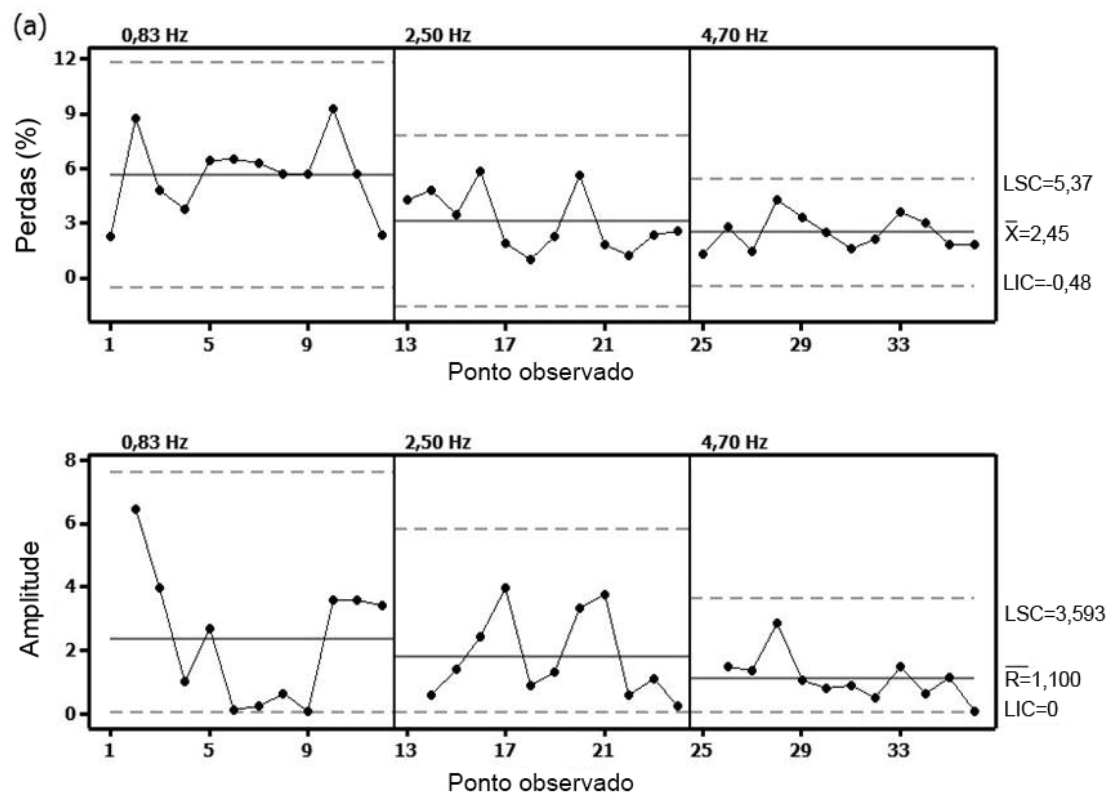

Figura 3. Carta de controle para perdas de frutos não destacados (\%) relacionadas à vibração (a) e carta de amplitude móvel (b) (Morrinhos, GO, 2012). LSC: limite superior de controle; LIC: limite inferior de controle; X e Amplitude: média. 
instabilidade para os maiores níveis de rotação avaliados, com distribuição de um valor acima dos limites de controle. Verificou-se que a amplitude entre os pontos 13-14 (12 rpm) e 27-28 (18 rpm) ultrapassaram o limite superior de controle, indicando, assim, que causas especiais interferiram no processo, refletindo nos pontos 13 e 26, os quais atingiram perdas totais superiores ao LSC (Figura 4b).

Analisando-se o efeito da rotação do sistema de trilha nas perdas referentes às ramas, foi possível verificar que a utilização de menores rotações proporcionou maiores perdas. Como não há trabalhos realizados com a cultura do tomate, tomou-se como parâmetro sistemas de trilhas radiais de colhedoras combinadas de grãos, pois essas também trilham e separam os grãos de partes vegetativas com a utilização da rotação.

Campos et al. (2005) observaram que as colhedoras de fluxo radial apresentam menores perdas na faixa de rotação de 800-900 rpm, quando comparadas com colhedoras radiais trabalhando com rotação do cilindro de 700-800 rpm, diferindo, assim, dos resultados obtidos por Costa et al. (2002), que encontraram maiores perdas na colheita de sementes de soja para maior rotação do cilindro trilhador. Entretanto, os autores citados, ao utilizarem maior rotação do cilindro, trabalharam com velocidade acima da recomendada para as colhedoras analisadas. A redução de perdas em rotações maiores do cilindro das colhedoras radiais se justifica pela maior dificuldade de trilha encontrada em rotações mais baixas, nessas colhedoras (Pinheiro Neto \& Gamero 1999).

Mesmo fora de controle, para as maiores rotações, é nítida a aproximação da maioria dos pontos da média, fato explicado pelos coeficientes de curtose e assimetria positivos. Logo, a presença de somente um ponto fora de controle mostra que a causa está relacionada à própria variabilidade do processo.

Outro fator inerente às perdas de frutos não recolhidos no solo foi a disposição de transplantio das mudas no solo, onde o espaçamento entre linhas foi de 1,2 m. De acordo com os produtores, o motivo da implantação da cultura nesses moldes foi a retirada da etapa de enleiramento, realizada de maneira manual. Outro fator também levado em consideração foi a velocidade operacional da colhedora, pois a massa a ser recolhida sem enleiramento (frutos e ramas) é menor do que a massa recolhida enleirada. Essa disposição de transplantio afeta as perdas de frutos no solo, em função da ramificação livre da planta, pois, fisiologicamente, o tomate para processamento industrial se desenvolve livremente pela superfície do solo. Sendo assim, as plantas podem se desenvolver em tamanhos que excedem a largura da plataforma de corte e recolhimento.

Como há variedades em que os frutos se desprendem com mais facilidade da rama, o processo de "arraste" da planta e seus frutos para a plataforma de

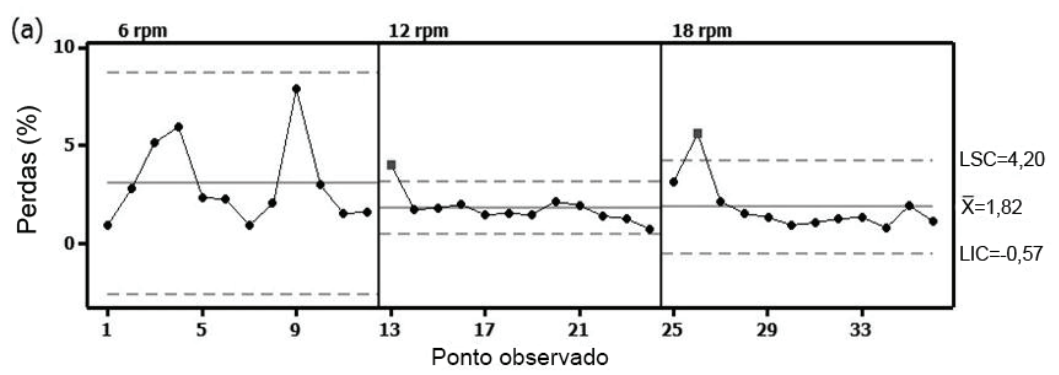

(b)

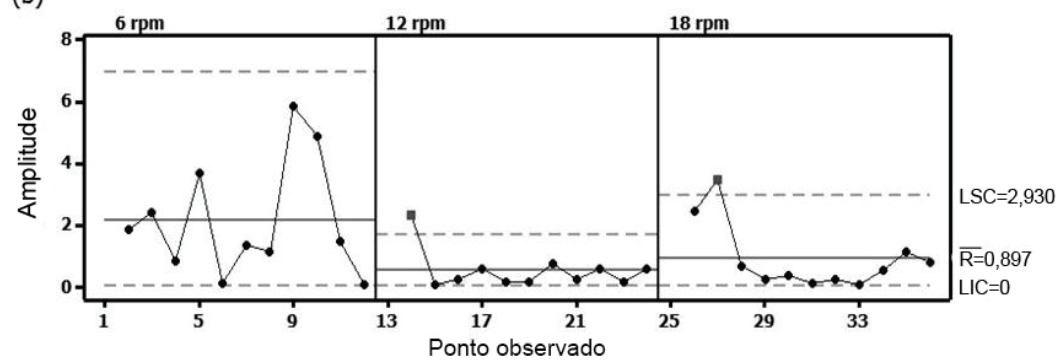

Figura 4. Carta de controle para perdas de frutos no solo (\%) relacionadas à rotação (a) e carta de amplitude móvel (b) (Morrinhos, GO, 2012). LSC: limite superior de controle; LIC: limite inferior de controle; X e Amplitude: média. 
corte pode ser comprometido pelo desprendimento dos frutos antes da entrada na plataforma. Em estudo realizado por Machado et al. (2011), a disposição do transplantio era em "blocos", sendo que havia uma distância de 0,6 m entre as linhas do bloco e 1,2 m entre os blocos. Para tal disposição do transplantio, há a necessidade do enleiramento, no qual as ramas livres são amontoadas com os frutos (desprendidos ou não de suas ramas) em carreadores da largura da plataforma de corte e recolhimento da colhedora, evitando, assim, o "arraste" de ramas livres para a máquina.

\section{CONCLUSÕES}

1. As perdas totais ocasionadas pela colhedora no sistema de separação apresentaram-se fora dos limites de controle e dos padrões aceitáveis de perdas, para a cultura do tomate industrial.

2. A adoção de maiores níveis de frequência de vibração $(4,7 \mathrm{~Hz})$ e de rotação (18 rpm) do sistema de separação proporcionaram menores perdas e, consequentemente, os melhores resultados.

3. A vibração influenciou nas perdas no sistema de separação da máquina, sendo necessária uma escolha criteriosa dos fatores frequência de vibração e rotação do sistema, durante a operação de colheita.

\section{REFERÊNCIAS}

ARAZURI, S.; ARANA, I.; JARÉN, C. Evaluation of mechanical tomato harvesting using wireless sensors. Sensors, Basel, v. 10, n. 12, p. 11126-11143, 2010.

ARAZURI, S. et al. Influence of mechanical harvest on the physical properties of processing tomato (Lycopersicon esculentum Mill.). Journal of Food Engineering, Londres, v. 80, n. 1, p. 190-198, 2007.

CAMPOS, M. A. O. et al. Perdas na colheita mecanizada de soja no Estado de Minas Gerais. Engenharia Agrícola, Jaboticabal, v. 25, n. 1, p. 207-213, 2005.

CHIODEROLI, C. A. et al. Perdas de grãos e distribuição de palha na colheita mecanizada de soja. Bragantia, Campinas, v. 71, n. 1, p. 112-121, 2012.

COMPAGNON, A. M. et al. Comparação entre métodos de perdas na colheita mecanizada de soja. Scientia Agropecuaria, Trujillo, v. 3, n. 3, p. 215-223, 2012.

CORTEZ, L. A. B. et al. Sistemas de colheita para frutas e hortaliças: oportunidades para sistemas semimecanizados. Revista Frutas \& Legumes, Lisboa, v. 12, n. 1, p. 26-29, 2002.
COSTA, N. P. et al. Avaliação da qualidade de sementes e grãos de soja provenientes da colheita mecanizada, em diferentes regiões do Brasil. Engenharia Agrícola, Jaboticabal, v. 22, n. 2, p. 211-19, 2002.

EMPRESA BRASILEIRA DE PESQUISA AGROPECUÁRIA (Embrapa). Centro Nacional de Ciência do Solo. Manual de métodos de análise de solo. Rio de Janeiro: Embrapa, 1997.

FERREIRA, M. D. et al. Avaliação física do tomate de mesa "romana" durante manuseio na pós-colheita. Engenharia Agrícola, Jaboticabal, v. 26, n. 1, p. 321-327, 2006.

FREDDI, O. S. et al. Produtividade do milho relacionada com a resistência mecânica à penetração do solo sob preparo convencional. Engenharia Agrícola, Jaboticabal, v. 26, n. 1, p. 113-121, 2006.

JARÉN, C. et al. Processing tomato mechanical harvesting cost evaluation. Acta Horticulturae, Leuven, v. 1, n. 758, p. 295-297, 2007.

MACHADO, T. A. et al. Efeitos da máquina. Revista Cultivar Máquinas, Pelotas, n. 110, p. 14-18, 2011.

OLIVEIRA, E. et al. Influência da vibração das hastes e da velocidade de deslocamento da colhedora no processo de colheita mecanizada do café. Engenharia Agrícola, Jaboticabal, v. 27, n. 3, p. 714-721, 2007.

PETERSON, D. L. Mechanical harvester for processing oranges. Applied Engineering in Agriculture, St. Joseph, v. 14, n. 5 , p. 455-458, 1998.

PINHEIRO NETO, R.; GAMERO, C. A. Efeito da colheita mecanizada nas perdas quantitativas de grãos de soja (Glycine max (L.) Merrill). Energia na Agricultura, Botucatu, v. 14, n. 1, p. 69-81, 1999.

SILVA, R. P. et al. Qualidade da colheita mecanizada de feijão (Phaseolus vulgaris) em dois sistemas de preparo do solo. Ciência Agronômica, Fortaleza, v. 44, n. 1, p. 6169, 2013.

TATLIDIL, F. F. et al. The effect of crop losses during preharvest and harvest periods on production costs in tomato production in the Ayas and Nallihan districts of Ankara province. Turkish Journal of Agriculture and Forestry, Ankara, v. 29, n. 6, p. 499-509, 2005.

TOLEDO, A. et al. Caracterização das perdas e distribuição de cobertura vegetal em colheita mecanizada de soja. Engenharia Agrícola, Jaboticabal, v. 28, n. 4, p. 710-719, 2008.

UBIERNA, C. V. et al. La agricultura de precision y las TIC's en la recolección mecanizada de tomate. Revista Vida Rural, Madrid, n. 312, p. 44-48, 2010.

WHITNEY, J. D. Field test results with mechanical harvesting equipment in Florida oranges. St. Joseph: ASAE, 1998. 